\title{
Energy-Efficient Virtual Machine Placement Algorithm
}

\author{
Bello Sururah Apinke, Gazali Abdulwakil Adekunle, and Aderounmu Ganiyu Adesola \\ Computer Science and Engineering Department, Obafemi Awolowo University, \\ Ile-Ife. Osun State, Nigeria
}

\begin{abstract}
Cloud datacentres are large datacentres with thousands of servers that consume excessive energy and have significant carbon footprints. The increasing cloud users, on the other hand, are demanding more services with better response time. Hence, resources allocation, power management as well as better service delivery to users are challenging tasks for cloud providers. The problem has been formulated as a Bin Packing problem and many algorithms have been proposed with the aim of attaining maximum throughput and minimum computation time in order to achieve an energy efficient datacentre. In this study, a new algorithm called Neighbour-Fit was proposed to address the aforementioned problem. A model based on the algorithm, for Virtual Machine (VM) allocation, was designed. A web-based simulator was also developed using HTML, CSS and PHP to simulate the proposed model. The proposed algorithm was benchmarked with five existing allocation algorithms using throughput and computation time. The Neighbour-Fit algorithm is about 90 percent faster than the Almost Worst-Fit, Best-Fit, First-Fit and Worst-Fit algorithms. Although the Next-Fit algorithm is about 20 percent faster than the Neighbour-Fit algorithm, Neighbour-Fit algorithm utilizes 4 percent less number of servers than the Next-Fit algorithm. This performance infers that the Neighbour-Fit algorithm with a moderate computational time and a high throughput optimizes server utilization. This in turn reduces the power consumed by the servers in cloud datacentres.
\end{abstract}

Index Terms - Cloud Computing, Datacentre, Energy Efficient, Neighbour-Fit, Placement Algorithm.

\section{INTRODUCTION}

The growth of cloud computing has led to the setting up of massive datacentres with thousands of servers which in turn has increased the datacentres energy consumption. The resource and power management at this scale becomes an issue because cloud providers are interested in effective utilization of datacentre resources. Effective utilization of computing resources in a cloud datacentres entails reducing the number of running servers which, in effect, will reduce the energy consumption and consequently the operational expenses.
The economy of datacentre, according to [1], depends on three major building blocks. These building blocks are the electricity supply, networking infrastructure and cooling resources. In addition to the major building blocks are the cost of physical space (an estate) that is required to host the building and equipment, the operational expenditures incurred that are related to personnel, software licenses, and equipment depreciation. Thus, the economy of a datacenter can be summarized as follows:

$$
\begin{array}{r}
\text { Cost }_{\text {total }}=\text { Cost }_{\text {estate }}+\text { Cost }_{\text {power }}+\text { Cost }_{\text {networking }} \\
+ \text { Cost }_{\text {cooling }}+\text { Cost }_{\text {operation }}
\end{array}
$$

Reference [2] further decomposed the cost of power in (1) into the cost of power consumed by servers plus the cost of power consumed by switches plus the cost of power consumed by storage. This is summarized as follows:

$$
\begin{aligned}
\text { Cost }_{\text {power_hardware }}=\text { Cost }_{\text {power_servers }} & + \text { Cost }_{\text {power_switches }} \\
& + \text { Cost } \\
& \text { power_storage }
\end{aligned}
$$

The cost of power consumed by servers is further decomposed into the addition of the cost of power consumed by the CPU, memory, disk, mainboard, and Network Interface Card (NIC). Equation 3 summarized the cost of power consumption by a server.

$$
\begin{aligned}
\text { Cost }_{\text {power_servers }}= & \text { Cost }_{\text {power_CPU }}+\text { Cost }_{\text {power_memory }} \\
& + \text { Cost }_{\text {power_disk }}+\text { Cost }_{\text {power_mainboard }} \\
& + \text { Cost }_{\text {power_NIC }}
\end{aligned}
$$

Reference [3] concluded that the CPU of a server consumes the most important amount of power and the relationship between power and CPU utilization is linear. The mathematical model is as shown in (4).

$$
\mathrm{P}=\left[\frac{c}{c}(1-\propto)+\propto\right] P_{p}
$$

$\mathrm{P}$ is total power consumption of a Physical Machine (PM) at time $t, P_{p}$ is peak power consumption, $c$ is the total number of cores required by the resident $\mathrm{VMs}, \mathrm{C}$ is the total number of 
cores of a PM and $\propto$ is the percentage of idle power versus the peak power (this is usually 50 percent in a typical PM).

Hence, the economy of a datacentre depends largely on CPU utilization. Therefore, effective CPU utilization will cut down the running cost of a datacenter.

The Server and Energy Efficiency report states that more than $15 \%$ of servers in cloud datacentres are running without being used actively on a daily basis [4]. The United State (U.S.) Environmental Protection Agency (EPA) reported that the energy consumed by datacentres has doubled in the period of 2000 and 2006 and estimated another two fold increase over the next few years if the servers are not used in an improved operational scenario [5]. According to the Greenpeace International, some datacentres (including Akamai, Amazon, Apple, Facebook, Google, HP, IBM, Microsoft, Twitter, and Yahoo) use as much electricity as 250,000 European homes. Figure 1 shows the electricity consumption of various countries and the datacentres.

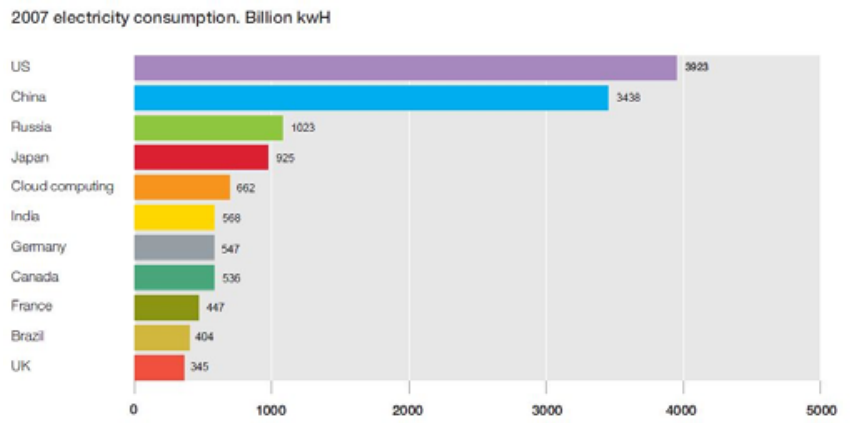

Fig. 1. Electricity consumption statistics of various countries in the year 2007. Source: [6].

It was found that if the cloud datacentres were a country its electricity demand would be more than the total electricity consumed by a big country like India, ranked 5 th in the world, and is expected to triple by 2020 [6].

Cloud users, on the other hand, are interested in minimizing service response time and optimizing overall application throughput [7]. It becomes a very challenging task in cloud environment to allocate the resources with minimum operating time along with effective utilization of available resources.

Scheduling is responsible for allocating servers in cloud datacentre to users' resource requests and it is at the heart of resource management. The basic unit of scheduling in cloud datacentres is Virtual Machine (VM), a software-defined computer. Users' resource requests are submitted in the form of VMs and the resources in cloud datacentre are allocated using a number of VM scheduling techniques. VM scheduling techniques is an on-going research. Its aim is to minimize running servers as well as time taken in carrying out their operations. However, existing models take long time in scheduling servers for $\mathrm{VM}$ requests in a cloud datacentre. Hence, there is the need to allocate VMs in the cloud environment within a minimum time and using a minimum number of servers.

\section{REVIEW OF LITERATURE}

Reference [8] explained that more than half of the electrical power in a datacenter is consumed by the IT loads (see Fig. 2). Servers consume $80 \%$ of the total IT load and $40 \%$ of total data center power consumption. The rest of power is consumed by other devices like transformers, distribution wiring, air conditioners, pumps, and lighting. Therefore the easiest and most obvious way to save energy is to run fewer PMs [9].

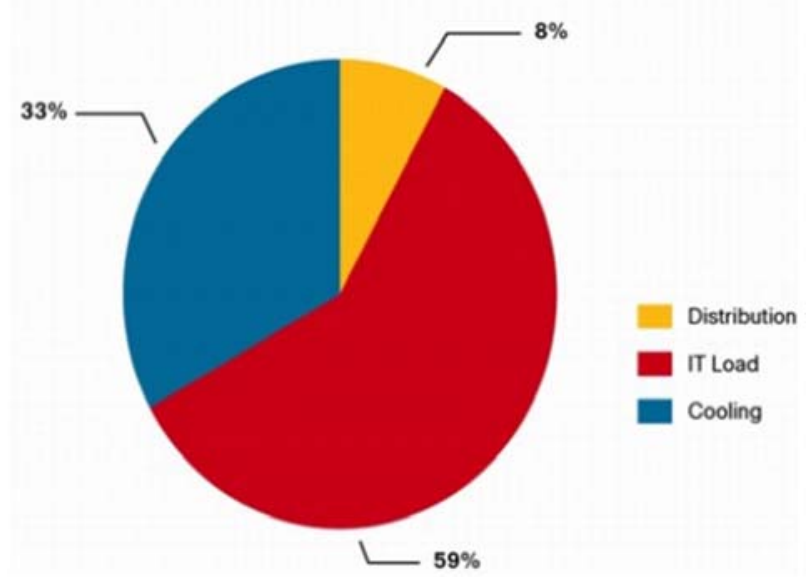

Fig. 2. Energy Consumption in a Datacentre.

Source:[8].

Cloud schedulers are implemented by placement algorithms. In recent years, several techniques like Constraint Programming, Integer Linear Programming, Genetics, Fuzzy, and Bin Packing techniques have been proposed for VMs placement in cloud datacentres [10]. Bin Packing technique is studied further in this work because of its usefulness in dynamic VM placement, especially where the demand is highly sporadic. Bin Packing is a heuristic based technique. It always generates a good solution in considerable amount of time. A Bin Packing technique is really useful when all PMs have the same amount of memory and processing capabilities.

Placement of VMs in a datacentre can be viewed as a Bin Packing problem. The PMs can be considered as bins having $n$ dimensions (CPU, RAM, and Disk). Similarly, the VMs can be considered as objects (having $n$ - dimensions) to be packed into these bins. These dimensions correspond to resource requirements and capacities of the VMs and PMs respectively. In Bin Packing problem, there is always the need to find a mapping between these objects and bins such that the total number of bins required is minimized. By applying this technique in datacentre, it is possible to minimize the cost of running datacentre by packing the VMs required to be running at a time onto the least number of PMs. Some attempts of heuristic Bin Packing algorithms proposed by several authors are explained below: 
Next-Fit algorithm, according to [11], places VMs in the order in which they arrive. It places the next VM in the request queue into the current PM if it fits. If it does not, leaves that PM, starts a new PM and then places the VM in it. For instance, assuming $S=\{4,8,5,1,7,6,1,4,2,2\}$ is a given set of VMs' CPU requests and CPU of datacentre's PMs are of size 10, placing the VMs into the PMs using Next Fit algorithm is shown in Fig. 3.

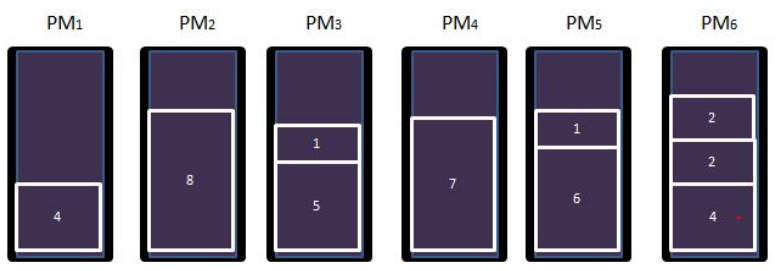

Fig. 3. VMs Placement under Next-Fit Algorithm.

The first request, four (4), was placed on $\mathrm{PM}_{1}$ leaving six as the remaining capacity of the PM. Eight (8) was checked against the remaining capacity of $\mathrm{PM}_{1}$ but the placement failed. Therefore, $\mathrm{PM}_{2}$ was started and became the current PM. The request, eight (8), was placed in this PM leaving two (2) as the remaining capacity. The new request, five (5), was placed in $\mathrm{PM}_{3}$ because $\mathrm{PM}_{2}$ can not accommodate it. The next request, one (1), was placed in $\mathrm{PM}_{3}$ because $\mathrm{PM}_{3}$ which was the current PM can accommodate it. Seven (7) is the next request. It was placed on $\mathrm{PM}_{4}$ because the remaining capacity on $\mathrm{PM}_{3}$ was four and is less than the request (7). $\mathrm{PM}_{5}$ becomes the current PM after six (6), the new request, has been placed on it. One (1) is placed on $\mathrm{PM}_{5}$ and $\mathrm{PM}_{5}$ remains the current $\mathrm{PM}$. The next request, four (4), was placed on $\mathrm{PM}_{6}$ because the remaining capacity on $\mathrm{PM}_{5}$ was three (3). The next request two (2) was placed on $\mathrm{PM}_{6}$ and the final request, two (2), in the queue was placed on $\mathrm{PM}_{6}$. At this point, $\mathrm{PM}_{6}$ remains the current $\mathrm{PM}$ because it still has two (2) unused capacities.

A total of six PMs are required to pack the VMs under Next Fit. This algorithm wastes PMs because some PMs may not be fully utilized (such as the first PM in Fig. 3). However, computation time for the Next-Fit algorithm is less because it does not perform any search during VM placement.

Reference [12] proposed an efficient VM assignment algorithm, the Resource-based First Fit Algorithm (RFFA), to assign VMs to PMs. Also dynamic placement of VMs to minimize SLA violations is studied in [13]. The authors modeled the problems as a Bin Packing problem and use the well-known First Fit algorithm to place the VMs to datacentre's PMs periodically.

First-Fit algorithm potentially assigns a VM to one of PMs with smaller identifiers. Therefore, the PMs with larger identifiers could be shut down and then the number of running PMs can be minimized. In another words, the algorithm places VMs in the order in which they arrive. It places the next item into the lowest numbered PM in which it fits. If it does not fit into any opened PM, it then starts a new PM. Figure 4 shows how First-Fit algorithm allocates VMs to PMs using the same set of VM requests and the same capacity of PMs as above.

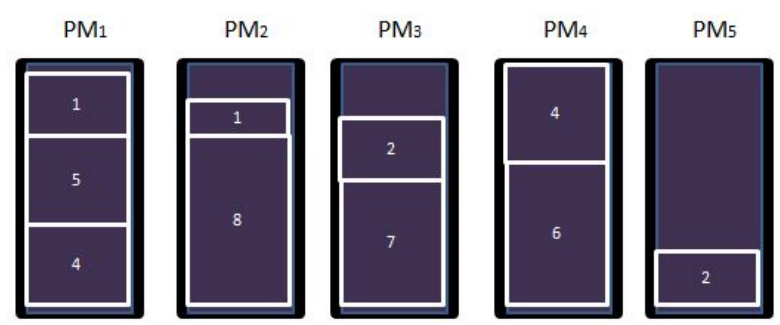

Fig. 4. VMs Placement under First-Fit Algorithm.

The first request, four (4), was placed on $\mathrm{PM}_{1}$ leaving six as the remaining capacity of the PM. Eight (8) was checked against the remaining capacity of $\mathrm{PM}_{1}$ but the placement failed. Therefore, $\mathrm{PM}_{2}$ was started and the request, eight (8), was placed in this PM leaving two (2) as the remaining capacity. The new request, five (5), was checked against the remaining capacity of $\mathrm{PM}_{1}$. The request, five (5) was placed on $\mathrm{PM}_{1}$ leaving one (1) as the remaining capacity. The next request, one (1), was checked against the remaining capacity of $\mathrm{PM}_{1}$. This request (1) was placed on $\mathrm{PM}_{1}$ leaving zero as the remaining capacity of $\mathrm{PM}_{1}$. The next request, seven (7), was checked against the remaining capacity of $\mathrm{PM}_{1}$ but the placement failed. Then, it was checked against the remaining capacity of $\mathrm{PM}_{2}$ but the placement failed. $\mathrm{PM}_{3}$ was started and the request was then checked against the remaining capacity. Seven (7) was then placed on $\mathrm{PM}_{3}$ leaving three (3) as the remaining capacity of $\mathrm{PM}_{3}$. The next request, six (6) was checked against the remaining capacities of $\mathrm{PM}_{1}, \mathrm{PM}_{2}$ and $\mathrm{PM}_{3}$ consecutively. The placement failed but $\mathrm{PM}_{4}$ was started and six (6) was placed on it. Four (4), the next request, was checked against the remaining capacities on $\mathrm{PM}_{1}, \mathrm{PM}_{2}, \mathrm{PM}_{3}$ and $\mathrm{PM}_{4}$ consecutively.

The placement of four failed on $\mathrm{PM}_{1}, \mathrm{PM}_{2}$ and $\mathrm{PM}_{3}$ but was successful on $\mathrm{PM}_{4}$ leaving no remaining capacity on $\mathrm{PM}_{4}$. The next request, one (1) was checked against the remaining capacities of $\mathrm{PM}_{1}$. The placement failed but when it was checked against the remaining capacity on $\mathrm{PM}_{2}$, One (1) was placed on $\mathrm{PM}_{2}$ leaving no remaining capacity on $\mathrm{PM}_{2}$. The next request, four (4), was checked against the remaining capacities on $\mathrm{PM}_{1}, \mathrm{PM}_{2}, \mathrm{PM}_{3}$ and $\mathrm{PM}_{4}$ consecutively. The placement of the request, four (4) failed on $\mathrm{PM}_{1}, \mathrm{PM}_{2}$ and $\mathrm{PM}_{3}$ but was successful on $\mathrm{PM}_{4}$ leaving no remaining capacity on $\mathrm{PM}_{4}$. Two (2), was then picked and checked against the remaining capacities on $\mathrm{PM}_{1}, \mathrm{PM}_{2}$ and $\mathrm{PM}_{3}$ consecutively. The placement failed on $\mathrm{PM}_{1}$ and $\mathrm{PM}_{2}$ but was successful on $\mathrm{PM}_{3}$ leaving one (1) as the remaining capacity on $\mathrm{PM}_{3}$. The last request, two (2), was checked against the remaining capacities on $\mathrm{PM}_{1}, \mathrm{PM}_{2}$, $\mathrm{PM}_{3}$ and $\mathrm{PM}_{4}$ consecutively. The placement failed, then a new PM was started and the request, two (2), was placed on it $\left(\mathrm{PM}_{5}\right)$. This algorithm uses less number of PMs compared to the Next Fit algorithm but its computation time is far more than the Next Fit algorithm. 
Reference [14] proposed Modified Best Fit Decreasing (MBFD) algorithms for resource allocation. This algorithm sorts all VMs in decreasing order of utilization and allocates each VM to a PM that provides the least increase of utilization due to this allocation. If a VM does not fit in any of the running PMs, a new PM is started. Figure 5 shows how Best-Fit Decreasing algorithm allocates VMs to PMs using the same set of VM requests and the same capacity of PMs as above.

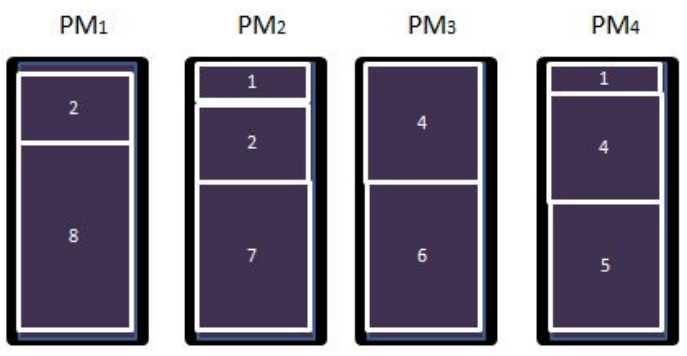

Fig. 5. Packing under Best-Fit Decreasing Algorithm.

The VM requests became $S=\{8,7,6,5,4,4,2,2,1,1\}$ after sorting. The first request, eight (8) was placed in $\mathrm{PM}_{1}$ leaving two (2) as the remaining capacity of the PM. The next request, seven (7) was checked against the remaining capacity on $\mathrm{PM}_{1}$ but the placement failed. $\mathrm{PM}_{2}$ was started the request was placed on it. The next request, six (6), was checked against the remaining capacities on $\mathrm{PM}_{1}$ and $\mathrm{PM}_{2}$. None of the PMs could accommodate the request. Therefore, $\mathrm{PM}_{3}$ was started and the request, six (6) was placed on it. The next request, five (5), was checked against the remaining capacities on $\mathrm{PM}_{1}, \mathrm{PM}_{2}$ and $\mathrm{PM}_{3}$. None of the PMs could accommodate the request. Therefore, $\mathrm{PM}_{4}$ was started and the request, five (5), was placed on it. The next request, four (4), was checked against the remaining capacities on $\mathrm{PM}_{1}, \mathrm{PM}_{2}, \mathrm{PM}_{3}$ and $\mathrm{PM}_{4}$. Only $\mathrm{PM}_{3}$ and $\mathrm{PM}_{4}$ could accommodate the request but $\mathrm{PM}_{3}$ would have the least remaining capacity after placement of the request. Therefore, the request, four (4), was placed on $\mathrm{PM}_{3}$ leaving no remaining capacity on it. The next request, four (4), was checked against the remaining capacities on $\mathrm{PM}_{1}, \mathrm{PM}_{2}, \mathrm{PM}_{3}$ and $\mathrm{PM}_{4}$ and only $\mathrm{PM}_{4}$ could accommodate the placement. Therefore, the request, four (4), was placed on $\mathrm{PM}_{4}$ leaving one (1) as the remaining capacity on it. The next request, two (2), was checked against the remaining capacities on $\mathrm{PM}_{1}, \mathrm{PM}_{2}$, $\mathrm{PM}_{3}$ and $\mathrm{PM}_{4}$. Only $\mathrm{PM}_{1}$ and $\mathrm{PM}_{2}$ could accommodate the request but $\mathrm{PM}_{1}$ would have the least remaining capacity after placement of the request. Therefore, the request, two (2), was placed on $\mathrm{PM}_{1}$ leaving no remaining capacity on it. The next request, two (2), was checked against the remaining capacities on $\mathrm{PM}_{1}, \mathrm{PM}_{2}, \mathrm{PM}_{3}$ and $\mathrm{PM}_{4}$ and only $\mathrm{PM}_{2}$ could accommodate the placement. Therefore, the request, two (2), was placed on $\mathrm{PM}_{2}$ leaving one (1) as the remaining capacity on it. The next request, one (1), was checked against the remaining capacities on $\mathrm{PM}_{1}, \mathrm{PM}_{2}, \mathrm{PM}_{3}$ and $\mathrm{PM}_{4}$. Only $\mathrm{PM}_{2}$ and $\mathrm{PM}_{4}$ could accommodate the request and would have the least remaining capacities after placement of the request. Therefore, the placement was done on $\mathrm{PM}_{2}$, being the $\mathrm{PM}$ with smaller identifier. The last request, one (1), was checked against the remaining capacities on $\mathrm{PM}_{1}, \mathrm{PM}_{2}, \mathrm{PM}_{3}$ and $\mathrm{PM}_{4}$ and only $\mathrm{PM}_{4}$ could accommodate the placement. Therefore, the request, one (1), was placed on $\mathrm{PM}_{4}$ leaving no remaining capacity on it.

Reference [15] has studied round robin algorithm to schedule and consolidate VMs. They have proposed a new strategy for VMs placement and migration that is called Dynamic RoundRobin (DRR). DDR as the extension of the Round-Robin method tries to reduce the number of active physical machines using two rules. In the first rule, if the running of a VM on a server has finished and there are still other VMs on the same physical machine, this physical machine will not accept new VMs.

In the second rule, if a physical machine remains in the first rule for a sufficiently long period of time, instead of waiting for the VMs to finish, the physical machine will be forced to migrate the rest of its VMs to other physical machines which in turn leads to shut down of physical machine after the migration completion.

Reference [16] proposed Most Full First algorithm. With this algorithm, PMs are sorted from most full to least full. Once sorting has been done, the VMs are allocated using First Fit.

Many of these placement/scheduling algorithms, apart from the Next-Fit and Round Robin algorithms, spend a considerable amount of time in sorting and searching before allocation of PMs to VMs is done. Also, the exempted algorithms allocate more PMs to VMs than other studied algorithms. Hence, this work intends producing a new heuristic (Neighbour-Fit) algorithm to strike the balance by allocating minimum number of PMs to VM requests and using minimum time.

\section{Methodology}

The activity diagram of the Virtual Machine allocation model as shown in Fig. 6 describes the movement of users' VM requests from the control node of the Cloud Providers to the pool of their servers (PMs).

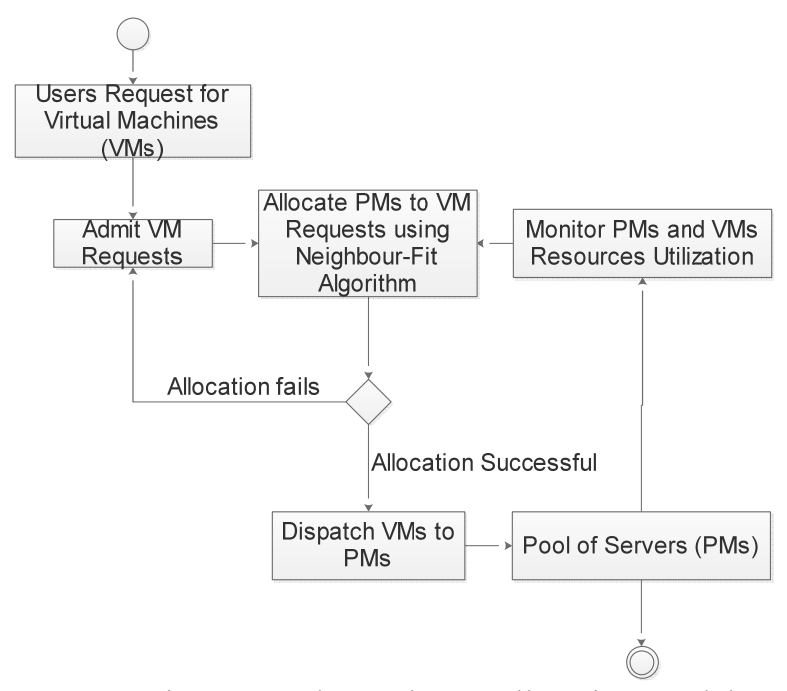

Fig. 6. On-demand VM Allocation Model. 
Generally, VM requests arrive at the cloud scheduler and admitted into a queue of requests. The requests are then checked for placement on the pool of PMs in the datacentre by the placement algorithm (in this study, the proposed Neighbour-Fit algorithm) using the information gathered at the monitoring module. If allocation is successful, the VM dispatcher creates the VMs on the PMs. If otherwise, the VM request is rejected and the algorithm proceeds to the next request in the queue.

Neighbour-Fit algorithm is a heuristic algorithm that can be used to address Bin Packing problems. It was inspired through the behaviour of the well known Next-Fit algorithms. Unlike the Next-Fit algorithm which places objects in the current bin if it fits or open a new (next) bin if otherwise, Neighbour-Fit algorithm places objects in the previous bin if it fit, and behaves like Next-Fit algorithm if otherwise. This behaviour is shown in Fig. 7.

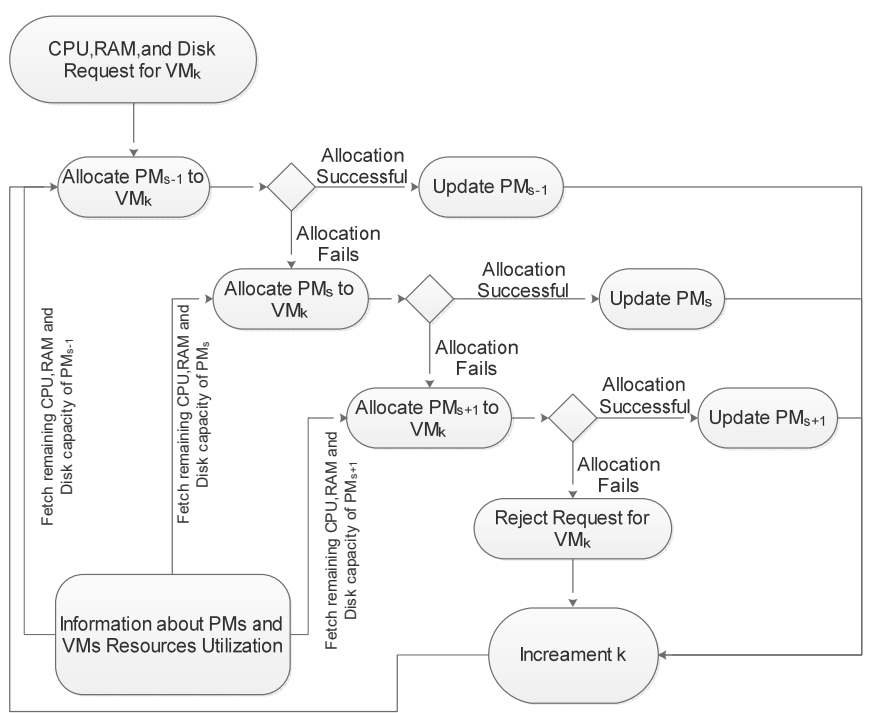

Fig. 7. Behaviour of Neighbour-Fit Algorithm in a Single Iteration.

Subscript $k$ and $s$ represent the current VM's request and current server (PM) respectively. The algorithm tries to allocate $\mathrm{PM}_{\mathrm{s}-1}$ to $\mathrm{VM}_{\mathrm{k}}$. If the allocation fails, it tries to allocate $\mathrm{PM}_{\mathrm{s}}$ to $\mathrm{VM}_{\mathrm{k}}$. If the allocation fails, it tries to allocate $\mathrm{PM}_{\mathrm{s}+1}$ to $\mathrm{VM}_{\mathrm{k}}$. If the allocation fails, it rejects the request $\left(\mathrm{VM}_{\mathrm{k}}\right)$ and picks another request by incrementing $k$ by one. Wherever the allocation is successful, the $\mathrm{PM}\left(\mathrm{PM}_{\mathrm{s}-1}, \mathrm{PM}_{\mathrm{s}}\right.$, or $\left.\mathrm{PM}_{\mathrm{s}+1}\right)$ is updated, made the current server and $k$ is incremented by one. The Neighbour-Fit algorithm and its sub function (bin filling function) is provided in Fig. 8 and Fig. 9 respectively.

Assuming $S=\{4,8,5,1,7,6,1,4,2,2\}$ is a given set of VMs' CPU requests and CPU of datacentre's PMs are of size 10, placing the VMs into the PMs using Neighbour-Fit algorithm is as shown in Fig. 10.

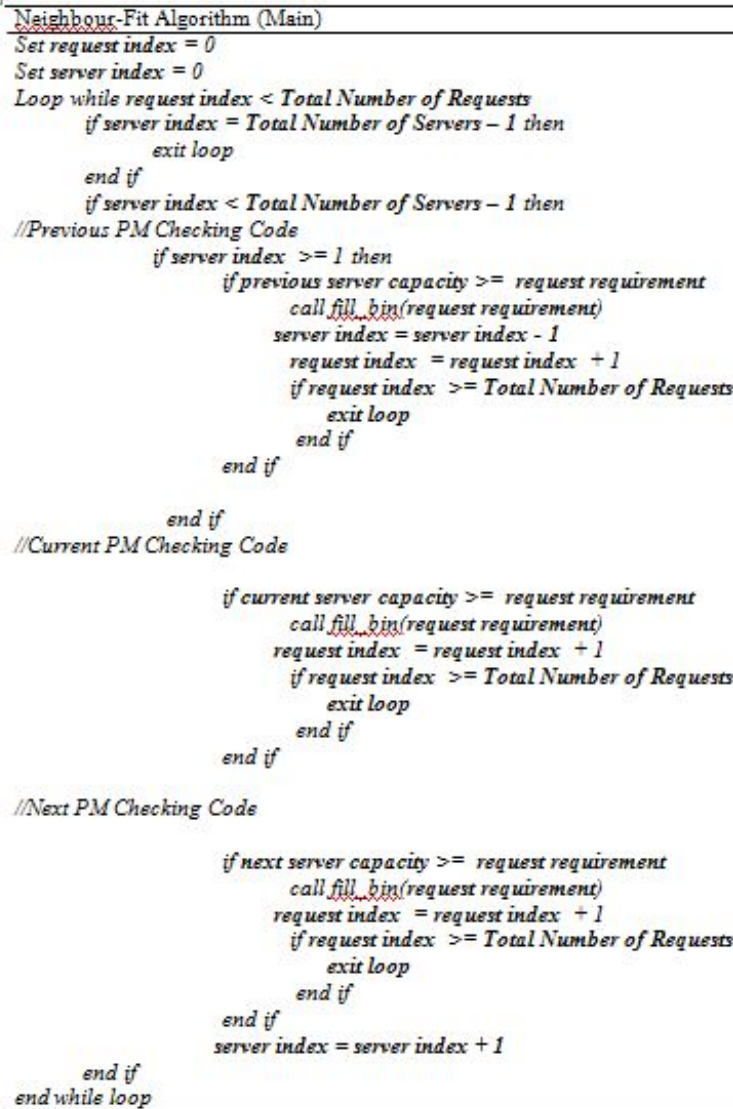

Fig. 8. Neighbour-Fit Algorithm.

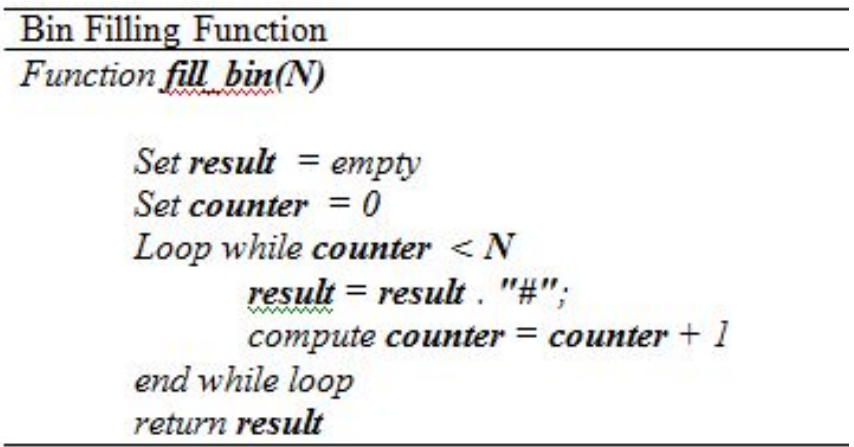

Fig. 9. Sub Function of Neighbour-Fit Algorithm.

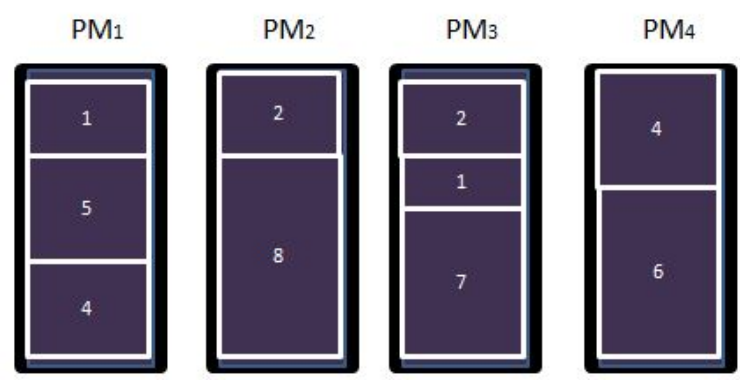

Fig. 10. Allocation Scenario According to Neighbour-Fit Algorithm. 
The first item in the request queue (4) is placed in $\mathrm{PM}_{1}$ leaving six (6) as the remaining capacity of $\mathrm{PM}_{1}$. The second item in the request queue (8) is higher than the remaining capacity on $\mathrm{PM}_{1}$ therefore, it is placed in $\mathrm{PM}_{2} . \mathrm{PM}_{2}$ became the current server. When the third item (5) is picked by the algorithm, it checked $\mathrm{PM}_{1}$ if it can accommodate the request. This item (5) is placed in $\mathrm{PM}_{1}$ leaving one (1) as the remaining capacity of $\mathrm{PM}_{1}$. The fourth item (1) is placed on $\mathrm{PM}_{1}$. The fifth item (7) is placed on $\mathrm{PM}_{3}$ because the remaining capacity on $\mathrm{PM}_{1}$ and $\mathrm{PM}_{2}$ are less than the current request. The sixth item (6) is placed on $\mathrm{PM}_{4}$ because the remaining capacity on $\mathrm{PM}_{3}$ is less than the current request. The seventh item (1) is placed on $\mathrm{PM}_{3}$ because the remaining capacity on $\mathrm{PM}_{3}$ was three (3) leaving two as the remaining capacity of $\mathrm{PM}_{3} . \mathrm{PM}_{3}$ becomes the current server. When the eight item (4) is picked by the algorithm, it checked $\mathrm{PM}_{2}$ if it can accommodate the request. The placement failed and the algorithm checked $\mathrm{PM}_{3}$. The placement also failed but successful when $\mathrm{PM}_{4}$ was checked. PM4 became the current server. When the ninth item (2) is picked by the algorithm, it checked $\mathrm{PM}_{3}$ if it can accommodate the request. The placement is successful and $\mathrm{PM}_{3}$ became the current server. When the tenth item (2) is picked by the algorithm, it checked $\mathrm{PM}_{2}$ if it can accommodate the request. The placement is successful and $\mathrm{PM}_{2}$ became the current server.

\section{A. Asymptotic Analysis of the Neighbour-Fit Algorithm}

Allocation is the mapping of PMs to VMs. It can be represented as shown in (5).

$$
\text { Allocation }=\text { Map }(\mathrm{PMs}, \mathrm{VMs})
$$

Each PM has three parameters $(C, R$ and $D)$. The $C, R$, and $D$ are the capacities (in percentage) of CPU, RAM, and Disk for each PM. Also, each VM has three parameters $(c, r$, and $d$ ). The $c, r, d$ are the requirements (in percentage) of CPU, RAM, and Disk for each VM request. The summation of $c$ must not equal or greater than the available $C$. The summation of $r$ must not equal or greater than the available $R$. Likewise, the summation of $d$ must not equal or greater than the available $D$. In addition to the previous conditions, the remaining capacities $\left(C_{s}^{\text {remaining }}, R_{s}^{\text {remaining }}\right.$, and $\left.D_{s}^{\text {remaining }}\right)$ of each PM can not be negative (that is, less than zero). These conditions are stated in (6) and (7).

$$
\begin{gathered}
\left\{\begin{array}{c}
\sum_{k}^{K} c_{k}<\sum_{s}^{S} C_{s} \\
\sum_{k}^{K} r_{k}<\sum_{s}^{S} R_{s} \\
\sum_{k}^{K} d_{k}<\sum_{s}^{S} D_{s}
\end{array}\right\} \\
\left\{\begin{array}{cc}
C_{s}^{\text {remaining }} \geq 0 \\
R_{s}^{\text {remaining }} \geq 0 \\
D_{s}^{\text {remaining }} \geq 0
\end{array}\right\}
\end{gathered}
$$

Assuming C, R, and D are initially 100 percent empty and are switched off except the first server (PM) in the array.

In the main Neighbour-Fit algorithm, $\mathrm{c}_{\mathrm{k}}, \mathrm{r}_{\mathrm{k}}$ and $\mathrm{d}_{\mathrm{k}}$ are compared with the $C_{s}^{\text {remaining }}, R_{s}^{\text {remaining }}$, and $D_{s}^{\text {remaining }}$ respectively. Whenever $C_{s}^{\text {remaining }}, R_{s}^{\text {remaining }}$, and $D_{s}^{\text {remaining }}$ are greater than the $c_{k}, r_{k}$ and $d_{k} ; c_{k}, r_{k}$ and $d_{k}$ values are then passed as argument into the function fill_bin. Function fill_bin has a loop that can iterate for the value of the argument (any of $c_{k}, r_{k}$ and $\mathrm{d}_{\mathrm{k}}$ ). Therefore, the maximum iteration of function fill_bin is the maximum of the VM requirements $\left(\mathrm{c}_{\mathrm{k}}, \mathrm{r}_{\mathrm{k}}\right.$ and $\left.\overline{\mathrm{d}_{\mathrm{k}}}\right)$ and is presented in (8).

$$
\mathrm{N}=\max (c, r, d)
$$

The number of iterations of Neighbour-Fit algorithm depends on the number of VM requests $(K)$ and the number of PMs $(S)$ in the datacenter. A loop that can iterate from zero to K-1 was constructed. Within the loop, statements that check for the attainments of $S$ were provided. If any of the statements returns true, the loop will terminate. Therefore, the number of iterations of Neighbour-Fit is the minimum of $K$ and $S$. This is presented in (9).

$$
\mathrm{M}=\min (K, S)
$$

Combining the two time complexities together, the asymptotic time complexity of Neighbour-Fit algorithm is in the order of $N^{*} M$ or $\mathrm{O}\left(N^{*} M\right)$.

\section{RESULT AND DISCUSSION}

The allocation algorithms under study were simulated 10 times with 2500,5000 , and $10000 \mathrm{VM}$ requests respectrively. In a single run of the simulation, for $2500 \mathrm{VM}$ requests, Fig. 11 shows the number of allocated PMs and Fig. 12 shows the computational time of each algorithm.

On the average, for $2500 \mathrm{VM}$ requests, the number of allocated PMs by each algorithm and the computational time of each algorithm were recorded as shown in Table 1 and Table 2 respectively. Neighbour-Fit, Almost Worst-Fit, Best-Fit and First-Fit algorithm allocated 111 PMs to the requests. Next-Fit algorithm allocated 115 PMs and Worst-Fit algorithm allocated $110 \mathrm{PMs}$ to the requests. Neighbour-Fit spent 0.33 second to allocate PMs to the requests. Almost Worst-Fit and Worst-Fit spent 3.58 second. Best-Fit and First-Fit spent 3.51 second and 2.38 second respectively. The Next-Fit algorithm spent 0.2 second to allocate PMs to the requests. Apart from the Next-Fit algorithm which was a little bit faster than the proposed algorithm (Neighbour-Fit), Neighbour-Fit proved to be significantly faster than other allocation algorithms. 


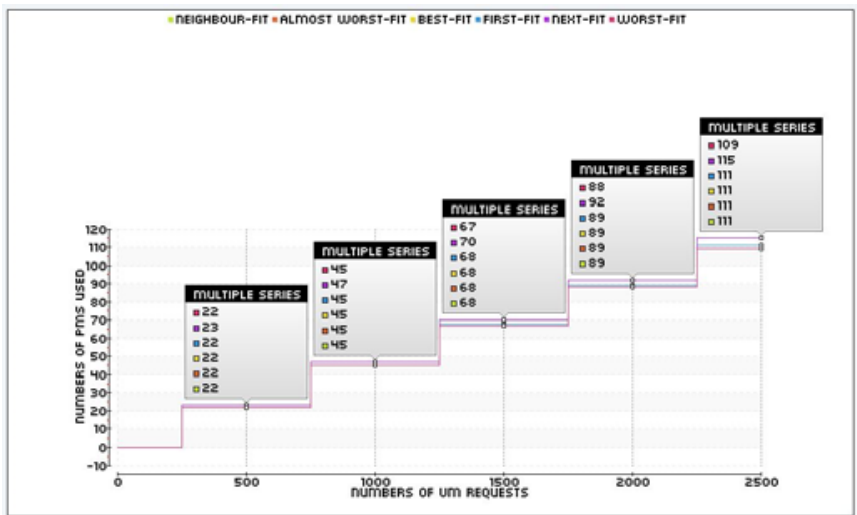

Fig. 11. Number of Allocated Servers for 2500 VM Requests at Intervals.

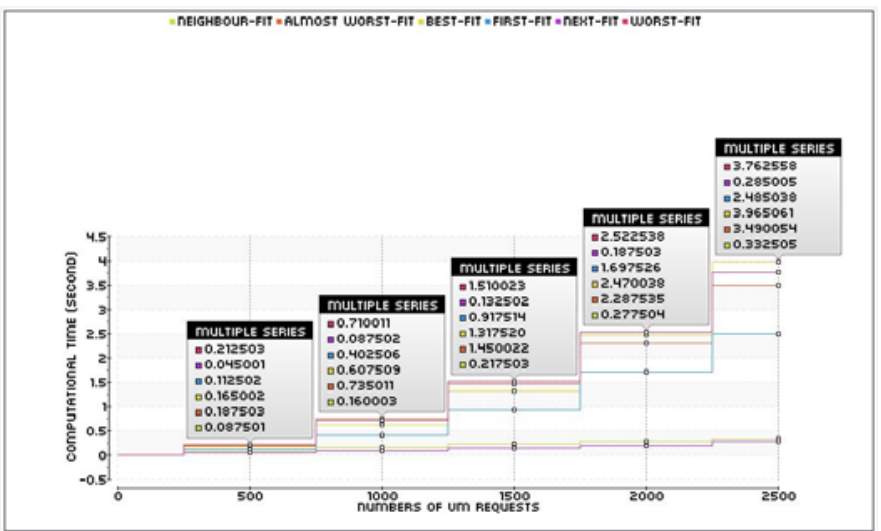

Fig. 12. Computation Time for 2500 VM Requests by Each Algorithm at Intervals.

Table 1. Number of Allocated PMs for 2500 VM Requests.

\begin{tabular}{|c|c|c|c|c|c|c|}
\hline Algorithms & $\begin{array}{l}\text { Neighbour- } \\
\text { Fit }\end{array}$ & $\begin{array}{l}\text { Almost Worst- } \\
\text { Fit }\end{array}$ & Best-Fit & First-Fit & Next-Fit & Worst-Fit \\
\hline \multicolumn{7}{|l|}{$\begin{array}{l}\text { Number of } \\
\text { Servers }\end{array}$} \\
\hline \multirow[t]{10}{*}{ (PMs) Used } & 110 & 111 & 111 & 111 & 115 & 108 \\
\hline & 111 & 111 & 111 & 111 & 117 & 110 \\
\hline & 111 & 111 & 111 & 111 & 116 & 110 \\
\hline & 111 & 111 & 111 & 111 & 116 & 110 \\
\hline & 111 & 111 & 111 & 111 & 116 & 110 \\
\hline & 110 & 111 & 111 & 111 & 115 & 109 \\
\hline & 111 & 111 & 111 & 111 & 115 & 110 \\
\hline & 111 & 111 & 111 & 111 & 115 & 110 \\
\hline & 110 & 110 & 110 & 110 & 115 & 110 \\
\hline & 111 & 111 & 111 & 111 & 114 & 109 \\
\hline $\begin{array}{c}\text { Mean } \\
\text { Standard }\end{array}$ & 110.7 & 110.9 & 110.9 & 110.9 & 115.4 & 109.6 \\
\hline Deviation & 0.483045892 & 0.316227766 & 0.31622777 & 0.31622777 & 0.843274043 & 0.699205899 \\
\hline
\end{tabular}

Table 2. Computational Time and Throughput of Each Algorithm for Placing 2500 VM Requests.

\begin{tabular}{ccccccc}
\hline Algorithms & $\begin{array}{c}\text { Neighbour- } \\
\text { Fit }\end{array}$ & $\begin{array}{c}\text { Almost Worst- } \\
\text { Fit }\end{array}$ & Best-Fit & First-Fit & Next-Fit & Worst-Fit \\
\hline $\begin{array}{c}\text { Computational } \\
\text { Time } \\
\text { (seconds) }\end{array}$ & 0.307018 & 3.749214 & 3.569204 & 2.357135 & 0.249014 & 3.548203 \\
& 0.328019 & 3.613207 & 3.753215 & 2.359135 & 0.278016 & 3.857221 \\
& 0.334019 & 3.999229 & 3.612206 & 2.392137 & 0.223012 & 3.472198 \\
& 0.320018 & 3.711212 & 3.398194 & 2.389136 & 0.225013 & 3.568204 \\
& 0.370021 & 3.402195 & 3.388194 & 2.482142 & 0.243014 & 3.4962 \\
& 0.294017 & 3.365192 & 3.403194 & 2.329134 & 0.36402 & 3.510201 \\
& 0.343019 & 3.368192 & 3.4992 & 2.43414 & 0.319019 & 3.948226 \\
& 0.34402 & 3.557204 & 3.4952 & 2.312132 & 0.224012 & 3.522201 \\
& 0.306017 & 3.553204 & 3.617206 & 2.303132 & 0.326018 & 3.438197 \\
$\begin{array}{c}\text { Mean } \\
\text { Standard } \\
\text { Deviation }\end{array}$ & $\mathbf{0 . 3 4 0 0 2}$ & 3.5082 & 3.367193 & 2.417139 & 0.219013 & 3.440197 \\
Throughput & $\mathbf{0 . 0 2 2 5 2 6 3 0 2}$ & $\mathbf{0 . 1 9 7 6 6 5 5 7 3}$ & $\mathbf{0 . 1 2 6 5 2 2 4}$ & $\mathbf{0 . 0 5 6 7 3 8 9 4}$ & $\mathbf{0 . 0 5 2 1 4 3 7 4 2}$ & $\mathbf{0 . 1 7 6 4 1 5 3 1 2}$ \\
(VM/s) & $\mathbf{7 6 0 7 . 5 9 8 8 3 5}$ & $\mathbf{6 9 7 . 7 9 6 7 9 5 9}$ & $\mathbf{7 1 2 . 1 8 9 7 2}$ & $\mathbf{1 0 5 1 . 5 0 8 7}$ & $\mathbf{9 3 6 2 . 7 6 6 3 7 5}$ & $\mathbf{6 9 8 . 3 0 3 5 8 0 4}$ \\
\hline
\end{tabular}

Throughput according to [17] is the number of VMs that are successfully allocated per time unit. Therefore, for $2500 \mathrm{VM}$ requests, the throughput of each allocation algorithm was calculated using (4) below and the results were also presented in Table 2.

$$
\text { Throughput }=\frac{\text { Number of allocated VMs }}{\text { Computational Time }}
$$

Neighbour-Fit had a throughput of $7607 \mathrm{VM} / \mathrm{s}$ which is significantly higher than other allocation algorithms under study except the Next-Fit. Although the Next-Fit algorithm is about 20 percent faster than the Neighbour-Fit algorithm, Neighbour-Fit algorithm utilizes 4 percent less number of servers than the Next-Fit algorithm. Figure 12 shows the throughput of each allocation algorithm.

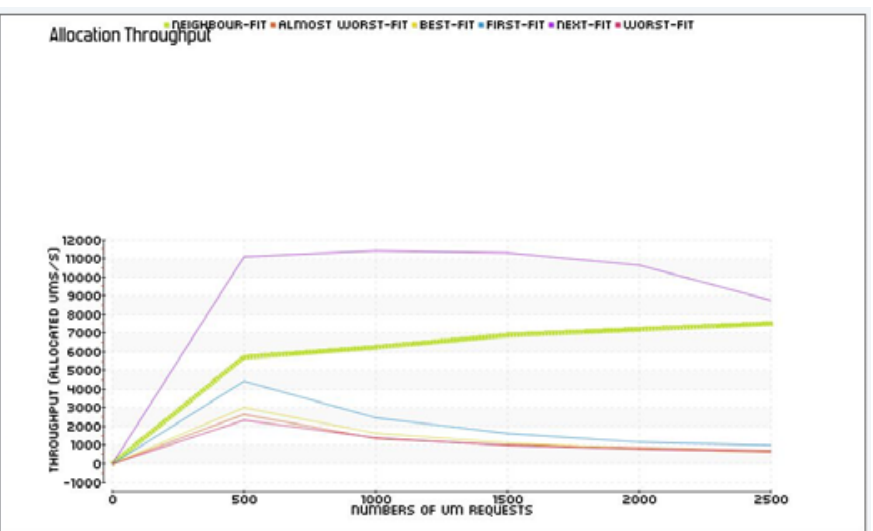

Fig. 13. Throughput of Each Allocation Algorithm.

\section{CONCLUSION}

In this study, a new VM placement algorithm (Neighbour-Fit) has been proposed. It is a step towards the implementation of a full-fledged cloud scheduler. It covers only the initial placement of VMs on a datacentre's PMs.

The results of the simulation have shown that the NeighbourFit algorithm is about 90 percent faster than the Almost WorstFit, Best-Fit, First-Fit and Worst-Fit algorithms. Although the Next-Fit algorithm is about 20 percent faster than the Neighbour-Fit algorithm, Neighbour-Fit algorithm utilizes 4 percent less number of servers than the Next-Fit algorithm. 
Therefore, the proposed algorithm is better than the other under-studied algorithms in terms of computational time and server utilization which in turn reduces power consumption of cloud datacentres. In the future, the algorithm could be implemented in an open-source cloud manager like Opennebula. Consolidation of PMs in cloud datacentres using the proposed algorithm will also be carried out.

\section{REFERENCES}

[1] D. P. Chandrakant, and J. S. Amip. (2005). 'Cost model for planning, development and operation of a data center'. Hewlett-Packard (HP), HP Laboratories, Palo Alto, CA, USA, Tech. Rep.

[2] L. Luo, W. Wu, D. Di, F. Zhang, Y. Yan, and Y. Mao. (2012). 'A resource scheduling algorithm of cloud computing based on energy efficient optimization methods'. In Green Computing Conference (IGCC), 2012 International (pp. 1-6). IEEE.

[3] X. Fan, W. D. Weber, and L. A. Barroso. (2007). 'Power provisioning for a warehouse-sized computer'. In ACM SIGARCH Computer Architecture News (Vol. 35, No. 2, pp. 13-23). ACM.

[4] S. Karayi. (2009). 'Server Energy and Efficiency Report'. Technical report, 1E, 2009.

[5] R. Brown, E. Masanet, B. Nordman, B. Tschudi, A. Shehabi, J. Stanley, J. Koomey, D. Sartor, and P. Chan. (2008). 'Report to Congress on Server and Data Center Energy Efficiency: Public Law 109-431'. Lawrence Berkeley National Laboratory, Berkeley, CA, USA, 2008. Pp14-15.

[6] G. Cook, and J. V. Horn. (2012). 'How dirty is your data? A Look at the Energy Choices That Power Cloud Computing'. Technical report, Green Peace International, April 20, 2012.

[7] V. S. Rathor, R. K. Pateriya, and R. K. Gupta. (2014). 'Survey on Load Balancing through Virtual Machine Scheduling in cloud computing Environment'. International Journal of Cloud Computing and Services Science (IJ-CLOSER). 3, 37-43.

[8] C. Ghribi. (2014). 'Energy efficient resource allocation in cloud computing environments'. Networking and Internet Architecture [cs.NI]. Institut National des T'el'ecommunications, 2014.

[9] C. Aschberger, and F. Halbrainer. (2013). 'Energy Efficiency in Cloud Computing'. $<$ http://www.uni-

salzburg.at/fileadmin/multimedia/SRC/docs/teaching/SS13/SaI/Paper_A schberger Halbrainer.pdf $>$ December 29, 2015.

[10] R. K. Gupta, and R. K. Pateriya. (2014). 'Survey on Virtual Machine Placement Techniques in Cloud Computing Environment'. International Journal on Cloud Computing: Services and Architecture (IJCCSA) , 4.

[11] K. Mills, J. Filliben, and C. Dabrowski. (2011). Comparing vm-placement algorithms for on-demand clouds. In Cloud Computing Technology and Science (CloudCom), 2011 IEEE Third International Conference on (pp. 91-98). IEEE.

[12] C. F. Kuo, T. H. Yeh, Y. F. Lu, and B. R. Chang. (2015). 'Efficient Allocation Algorithm for Virtual Machines in Cloud Computing Systems'. In Proceedings of the ASE BigData and SocialInformatics 2015 (p. 48). ACM.

[13] N. Bobroff, A. Kochut, and K. Beaty. (2007). 'Dynamic Placement of Virtual Machines for Managing SLA Violations,' in Proc. of the IFIP/IEEE International Symposium on Integrated Network Management (IM'07), 2007.

[14] R. Buyya, A. Beloglazov, and J. Abawajy. (2010). 'Energy-efficient management of data center resources for cloud computing: a vision, architectural elements, and open challenges'.arXiv preprint arXiv:1006.0308.

[15] C. C. Lin, P. Liu, and J. J. Wu. (2011). 'Energy-efficient virtual machine provision algorithms for cloud systems'. In Utility and Cloud Computing (UCC), 2011 Fourth IEEE International Conference on (pp. 81-88). IEEE.

[16] S. Lee, R. Panigrahy, V. Prabhakaran, V. Ramasubramanian, K. Talwar, L. Uyeda, and U. Wieder. (2011). 'Validating Heuristics for Virtual Machines Consolidation. Microsoft Research MSR-TR-2011-9. pp. 1-14.

[17] S. Subramanian, N. G. Krishna, K. M. Kumar, P. Sreesh, and G. R. Karpagam. (2012). 'An adaptive algorithm for dynamic priority based virtual machine scheduling in cloud'. International Journal of Computer Science Issues(IJCSI), 9(6).

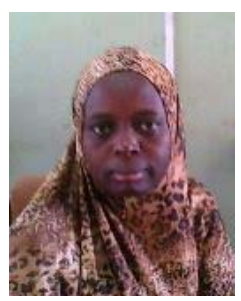

SURURAH Bello obtained B.Sc. Computer Engineering in 1995, M.Sc. Computer Science in 2002 and Ph.D Computer Science in 2011 from the Department of Computer Science and Engineering, Obafemi Awolowo University, Ile-Ife, Nigeria. She is currently a Senior Lecturer in the same department. Her research interests include Distributed Computing, Artificial Intelligence and Social Computing.

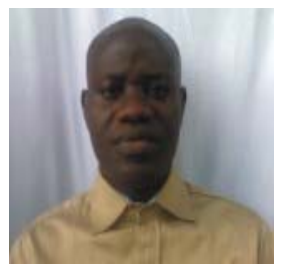

GAZALI Abdulwakil. Adekunle. was born in Lagos State, Nigeria, in 1981. He received the B.Sc. in Computer Engineering and M.Sc Computer Science from Obafemi Awolowo University, IleIfe, Osun State, Nigeria in 2012 and 2016 respectively.

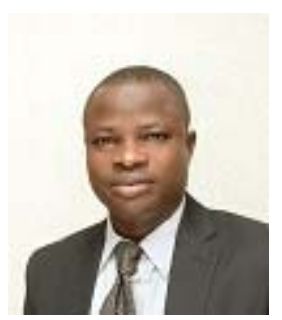

Prof. ADEROUNMU Ganiyu Adesola is a Professor of Computer Science and Engineering in Obafemi Awolowo University, Ile-Ife. He is the current Director, Information Technology and Communications Unit, Obafemi Awolwo University, Ile-Ife, Nigeria. Professor Aderounmu received his B.Sc. Degree in Computer Engineering and M.Sc. Degree in Computer Science from Obafemi Awolowo University, Ile-Ife in 1991 and 1997 respectively. He is the current President, Nigeria Computer Society. Professor Aderounmu is a visiting research fellow, University of Zululand, Republic of South Africa. 